\title{
EDITORIAL
}

\section{IN THE JANUARY 2010 ISSUE OF CLINICS}

\author{
Mauricio Rocha-e-Silva, Editor
}

doi: 10.1590/S1807-59322010000100001

As Clinics opens its sixth year of existence, its second as a monthly periodical, we shall be expecting our first posting of an Impact Factor by the ISI Journal of Citations report. Over this period, CLINICS gradually evolved from a publisher of articles originating mainly from the Medical College of Sao Paulo University to a more cosmopolitan Brazilian and International publisher of science. This trend, illustrated in Fig. 1, strongly suggests that the posting of our first impact factor will enhance the trend towards a truly cosmopolitan journal.

In this issue, we publish eight Clinical Science reports.

Hovnanian et al. endeavored to determine long-term survival, identify preoperative factors predictive of a favorable outcome, and assess functional improvement after coronary artery bypass grafting in 244 patients with advanced left ventricular dysfunction and found that for selected patients with severe ischemic left ventricular dysfunction and predominance of tissue viability, coronary artery bypass grafting it may be possible to implement preoperative clinical/ functional parameter measurements for predicting outcome, such as left ventricular ejection fraction and gated left ventricular ejection fraction at exercise or at rest.

Tiryakioglu et al. examined the frequency of Cushing's syndrome (CS) in 150 obese patients devoid of specific clinical symptoms of Cushing's syndrome and found that a significant proportion $(9.33 \%)$ of patients with simple obe-

Hospital das Clínicas, Faculdade de Medicina da Universidade de São Paulo - São Paulo/SP, Brazil.

mrsilva36@hcnet.usp.br sity had Cushing's syndrome; they argue that obese patients should be routinely screened for Cushing's syndrome.

Barbosa et al. propose to improve the transmission of information between requiring physicians and radiologists by means of a structured report for thyroid sonography developed according to information gathered from radiologists and endocrinologists working in the field. They found that implementation of the structured report in a university hospital was viable, and that radiologists and endocrinologists preferred the structured report when compared to free text, and both agreed that the former improved the transmission of information.

Dantas et al. evaluated the kinetics with which a cholesterol-rich emulsion (called a low-density emulsion) binds to low-density lipoprotein receptors in a group of 10 patients with grade III obesity, versus 10 non-obese healthy control individuals by the fractional clearance rate, and found that grade III obese subjects exhibited normal low-density lipo- 
protein removal from plasma as tested by the nanoemulsion method, but triglyceride removal was slower.

Horasanli et al. compared the efficacy of combined lumbar plexus-sciatic nerve blocks versus epidural anesthesia in 40 patients per group undergoing total knee surgery, and found that the lumbar plexus-sciatic nerve blocks provide effective unilateral anesthesia and may offer a beneficial alternative to epidural anesthesia in patients undergoing total kneesurgery.

Scorleze-Ferrer et al. examined causes of admission in the public health system for children from zero to nine years of age in the city of São Paulo during the years 2002 to 2006 and compared these results to those from the national data, and found a paradoxical increase in the number of hospitalizations during an expansion of primary attention, indicating that the rise was not associated with a significant improvement in the quality of service.

Ramos et al. determined differences in cardiac vagal tone values in non-obese healthy, adult men with and without unfavorable anthropometric characteristics, and found that non-obese and healthy adult men with unfavorable anthropometric characteristics tend to present lower cardiac vagal tone levels. They claim that early identification of this trend by simple protocols that are non-invasive and risk-free, using select anthropometric characteristics, may be clinically useful in a global strategy to prevent cardiovascular disease.

Melotti et al. evaluated the value and limitations of clonality analysis in the diagnosis of primary cutaneous B-cell lymphomas (PCBCL) and B-cell pseudolymphomas (B-PSL). The study included 29 cases of B-cell lymphoproliferative processes classified as primary cutaneous B-cell lymphomas (13), B-PSL (6) and inconclusive cases (10) using histology and immunohistochemistry, and concludes that the use of the two protocols, IgH FR3-trad and IgL-Kappa (IgL-K) Biomed protocols for clonality analysis improved diagnostic accuracy.
We also publish four Basic Science papers

Morais Silva et al. analyzed the effects of fluoxetine, a selective serotonin re-uptake inhibitor, on the enamel organ of rats and found no structural changes in the experimental group compared to the controls, suggesting that, at the dose used, fluoxetine did not interfere with serotonin-mediated development of the enamel organ or the process of amelogenesis.

Sallum et al. evaluated transcapillary refill in a model of retroperitoneal uncontrolled, treated hemorrhage in dogs and concluded that despite the rebleeding observed in treated groups, the utilization of hypertonic saline solution with dextran proved to be effective in the initial reanimation, producing evident transcapillary refill, while the Lactated Ringer's solution produced capillary extravasation and was ineffective in the initial volume replacement in this model of uncontrolled hemorrhage.

Subhash et al. describe the normal and variant anatomy of the coronary artery ostia in Indian subjects and find that the preferential location of the ostia was within the sinus and above the cusps, but below the sinutubular ridge. On occasion, normal variants such as multiple ostia, vertical or circumferential shift in the position, and slit-like ostia may create confusion in interpreting the images and pose a difficulty during procedures like angiography, angioplasty, and coronary artery bypass grafting.

Norhazlia et al. describe the effect of eurycoma longifolia on spermatogenesis in estrogen-treated adult male rats and claim that the extract acts as a potential agent for reversing the effects of estrogen by increasing spermatogenesis and sperm counts in rats after fourteen consecutive days of treatment.

We also publish a review on coronary heart disease in women and 3 case reports. 